\title{
Development and validation of a loop mediated isothermal amplification assay to detect severe acute respiratory syndrome corona virus-2
}

\author{
BN Iqbal $^{1}$, S Arunasalam ${ }^{1}$, MVM Divarathna ${ }^{1}$, AA Jabeer ${ }^{1}$, UTN Senaratne ${ }^{2}$, \\ PDNN Sirisena $^{3}$, R Muthugala ${ }^{4}$, F Noordeen ${ }^{1 *}$
}

Introduction and Objectives:The ability to detect SARS-CoV-2 using an alternativesimple molecular assayduring the pandemic is crucial for the mitigation efforts in low / middle income countries. We have developed and validated a rapid and simple loop mediated isothermal amplification assay (LAMP) for screening of SARS-CoV-2 infection in known infected and non-infected individuals.

Methods: Sixsets of primers were designed targeting the N-gene of the SARS-CoV-2 (Accession ID MN994468) using the Primer Explorer software V5. LAMP reactions were performed using Warm Start 2X Master Mix and real-time PCR machine at $65^{\circ} \mathrm{C}$ for 60 cycles with 15 seconds for each cycle. Results were read by visualizing turbidity under ultraviolet (UV) light and real time fluorescence detection through FAM channel of the real time PCR machine and compared with the results obtained from the real time reverse transcription polymerase chain reaction (rtRT-PCR) (Altona Diagnostics, Germany).We tested 230 SARS CoV-2 positive (Ct values $<25)$ and 20 SARS CoV-2 negative samples by rtRT-PCR using the newly developed LAMP assay.

Results: A total of 200 of 230 SARS CoV-2 positive samples tested positive with the newly developed LAMP assay with a sensitivity of $87 \%$. All 20 SARS CoV-2 negative samples were tested negative by the newLAMP assay with a specificity of $100 \%$.

Conclusions: The newly developed LAMP assay has a sensitivity of $87 \%$ and specificity of $100 \%$ compared with the rtRT-PCR for the detection of SARS CoV-2. We will be testing more samples to strengthen our findings with cost analysis for promoting for wider use.

Key words: SARS-CoV-2, Loop mediated isothermal amplification, Sensitivity, Specificity

Funding: National Research Council’s Rapid response to COVID-19 (NRC-CVD 12-20).

\footnotetext{
${ }^{1}$ Department of Microbiology, Faculty of Medicine, University of Peradeniya, Sri Lanka

${ }^{2}$ Department of Multidisciplinary Sciences, Faculty of Allied Health Sciences, General Sir John Kotelawala

Defense University, Ratmalana, Sri Lanka

${ }^{3}$ ImmunifyMe Health Tech, New Delhi, India

${ }^{4}$ National Hospital, Kandy, Sri Lanka

Address for correspondence:BN Iqbal. Telephone: +94770333687 Email: iqbalbushran@gmail.com

iD https://orcid.org/0000-0002-6299-9556
} 